\title{
Dark Side Traits at Work: Bright and Dark Side Traits and Job Value Preferences
}

\author{
Adrian Furnham1,2, David Pendleton 3,4 \\ ${ }^{1}$ Department of Clinical, Educational, and Health Psychology, University College London, London, UK \\ ${ }^{2}$ Norwegian Business School (BI), Olso, Norway \\ ${ }^{3}$ Edgecumbe Consulting, Bristol, UK \\ ${ }^{4}$ Said Business School, Oxford University, Oxford, UK \\ Email: a.furnham@ucl.ac.uk
}

Received 4 April 2016; accepted 16 May 2016; published 19 May 2016

Copyright (C) 2016 by authors and Scientific Research Publishing Inc.

This work is licensed under the Creative Commons Attribution International License (CC BY).

http://creativecommons.org/licenses/by/4.0/

c) (i) Open Access

\begin{abstract}
Two hundred and twenty-one business executives completed the Big Five NEO-RI-R (Costa \& McCrae, 1992; an inventory of normal personality), the Hogan Development Survey (HDS) (Hogan \& Hogan, 1997; an inventory of career derailing tendencies) and the Motives, Values and Preferences Inventory (MVPI) (Hogan \& Hogan, 1999; an inventory of ten core occupational values). Correlational and regression analyses revealed modest but predictable relationships between all personality traits (except Neuroticism) and the values, particularly Recognition, Power and Security. The personality traits accounted for most variance with respect to Aesthetics, Altruism, Security and Power. Correlations and regressions for the HDS and MVPI showed that the values most related to "flawed interpersonal style" were Recognition, Affiliation, Power and Security. Stepwise regressions indicated that the HDS accounted for incremental validity over the Big Five in predicting core work values. Implications of these findings were considered.
\end{abstract}

\section{Keywords}

Dark Side Personality

\section{Introduction}

This paper is concerned with two aspects of personality as well as specific motives and preferences. The two aspects of personality are first the "bright-side" normal personality variables derived from the now widely accepted Five Factor Model, while second the "dark-side" abnormal personality variables based on ideas from the psychiatric attempts to classify the personality disorders. 
This paper examines how normal "bright side" (Big Five) and abnormal "dark side” (Hogan Development Survey) personality traits relate to core (work) values. Over the past decade, there have been a number of studies based on differential psychology and personality disorder psychiatry (De Clercq \& De Fruyt, 2003; Stepp \& Trull, 2007; Widiger, Costa, \& McCrae, 2001; Widiger, Trull, Clarkin, Sanderson, \& Costa, 2002). There is now a very interesting and growing literature on the personality disorders at work looking at such things as "successful psychopaths" (Babiak \& Hare, 2006) to how each disorder is related to particularly patterns of derailment in the workplace (Furnham, 2007; Kets de Vries, 2006, 2007; Kets de Vries \& Miller, 1985).

There is also a growing literature on the relationship between dark side variables and work success and failure (Furnham, Hyde, \& Trickey, 2013, 2014a, 2014b; Gaddis \& Foster, 2015; Judge, Piccolo, \& Kosalka, 2009; Kaiser, Hogan, \& Craig, 2008; Kaiser, Craig, Overfield, \& Yarborough, 2011; Kaiser \& Craig, 2011).

Hogan (2006) distinguishes three further aspects of personality, referred to as "the bright side", "the dark side", and "the inside". The bright side reflects peoples' performance when they are acting normally in day-today situations; inventories of normal personality concern the bright side. The Big Five Model is an accepted taxonomy of the bright side. The dark side reflects peoples' performance when they are tired, stressed, or otherwise not paying attention. The spectrum hypothesis suggests that dark-side behaviours are manifestations of extreme (high or low) scores on normal personality traits. Dark side tendencies derail careers; the DSM-IV, Axis 2 personality disorders provides a taxonomy of these tendencies (Furnham \& Taylor, 2004; Hogan, 2006). The inside reflects peoples' core values. There is still no real consensus regarding a taxonomy of core values. Hogan and Hogan (1999) proposed the Motives, Values, and Preferences Inventory (MVPI) as a tentative taxonomy of occupational values, which will be used in this study.

There have been some attempts to relate the two worlds of traits and disorders (Dyce, 1997) from the two disciplines of psychology and psychiatry. Wiggins \& Pincus (1989) note that the personality disorders are "readily interpretable” (p.314) within the Big Five framework. Perhaps the most comprehensive comparative attempt has been made by Widiger et al. (Widiger \& Coker, 2001; Widiger \& Costa, 2001; Widiger et al., 2001; Widiger et al., 2002), who believe that having extreme (high or low) scores on personality traits renders individuals at risk for certain disorders. Thus very high conscientiousness may be a marker for obsessiveness, while very high introversion scores are markers for the Schizoid personality disorder.

Thus, the spectrum hypothesis suggests considerable correlation between measures of traits (bright side) and disorders (dark side) although not all traits (in the Big Five or Big Seven systems) would be related to the disorders. Certainly one may predict that disorders like being highly reserved (i.e., extremely introverted) would show very strong negative association with a value like Affiliation. Similarly whilst the bright side traits may not be strongly correlated with Recognition specific dark side traits like Colourful (Narcissistic) would be. More importantly where bright and dark side traits are regressed onto values particularly Recognition, Power and Hedonism it is probable there would be considerable incremental validity of the latter dark side traits over the former.

Rolland \& De Fruyt (2003) were the first to study the "bright and dark side" in conjunction in a job-related context. In a study of 130 French military, they found that personality disorders (labelled maladaptive traits) did not predict negative affect beyond the Big Five personality traits, suggesting that they have no incremental validity in predicting affective (military) work outcomes. On the basis of studies in the manual (Hogan \& Hogan, 1999) it was predicted that Bold (narcissistic) would be related to valuing Power, Reserved (Schizoid) to not valuing Affiliation; Diligent (Obsessive) and Dutiful (Dependent) valuing Tradition and Imaginative (Schizotypal) valuing Aesthetics.

This study examines the relationship of the bright side and dark side personality variables and salient work related values. It replicates and extends previous work in this field (Furnham, Trickey, \& Hyde, 2013). The central question of the study is the extent to which a person's values can be inferred from (or overlap with) their bright and dark side profiles. It has been argued that traits and values represent two fundamentally different aspects of personality (McAdams, 1995; Winter, John, Stewart, Klohnen, \& Duncan, 1998). Motives and values relate to goals, desires and wishes, whereas traits concern how people typically behave. Thus introverts and extraverts may pursue the same goal in systematically different ways. For instance introverted academics may seek to gain Recognition by primarily published works while extroverted academics by conference and media appearances. Equally introverts and extraverts may seek and wield Power quite differently - the former through legal or organisational systems, the latter by personal contact.

Extreme extraverts and introverts may also behave quite differently under stress and external pressure. This is where the dark side disorders linked to Extraversion (i.e., Excitable, Colourful) may be relevant. Thus Colourful 
individuals under stress may adopt partly adaptive (help seeking) and partly maladaptive (Hedonism) coping strategies linked to their fundamental values which reflect their goals, desires and wishes.

This study will examine the relationship between widely accepted trait models (both normal range, i.e., personality traits and extreme, i.e., personality disorder) and an increasingly widely used parsimonious and theoretically based model of motives and values. One central issue concerns conceptual and statistical overlap between these instruments and hence the extent to which they are interchangeable or redundant. Little overlap between tests measuring the same thing can be ascribed to the tests having poor validity. However, all three measures used here have ample evidence of nearly all types of validity (concurrent, construct, predictive). Hence the issue is which dimensions correlate and which not, to what degree and why. This is a particularly salient issue for applied psychologists eager to do a thorough person assessment but constrained by time.

There are many taxonomies of values and value systems in the area of personality and vocational psychology. This study will use the Hogan \& Hogan's (1997) Motives, Values, Preferences Inventory (MVPI), which is a modern, psychometrically valid measure with impressive adult norms. It measures 10 values set out in Table 1. It is based on Holland's work and ideas (Hogan \& Blake, 1999), which will be described in due course. Inevitably there have been various attempts to examine the relationship between personality measures and values mainly using the Holland measure (Costa, McCrae, \& Holland, 1984; De Fruyt \& Mervielde, 1997). This study examined the relationship between the Big Five and the ten values in the MVPI. We predicted that four of the five Big Five factors (that is all except Neuroticism) would be correlated with values.

Following from the studies reported in the manual and the theory behind both the trait and values measures we predicted that Extraversion would be highly positively correlated with Recognition and Affiliation; that is, extraverts want fame, power, fun, friends, and enjoy taking risks. We also predicted that Openness would correlate with Aesthetics; open people are interested in form, style, and design issues. We then predicted that Agreeableness would correlate with Altruism; agreeable people are interested in finding a better world for the unfortunate, but not interested in careers or money. We then predicted that Conscientiousness would be correlated with Tradition and Security; conscientious people want predictability, stability, and order.

\section{Method}

\subsection{Participants}

There were 221 participants of which 175 were male. Their ages ranged from 21 to 60 years with a median of 43 years and a mean of 41.71 years. With 2 exceptions they were all native white Britons. All were university graduates. They were all at a managerial level in a large, mostly multi-national organisation. On average they had had around 15 years working experience.

\subsection{Measures}

Personality. The NEO Personality Inventory-Revised (NEO-PI-R; Costa \& McCrae, 1992). This 240-item, non-timed questionnaire measures 30 primary personality traits (facets) and the underlying 'Big Five' personality factors (domains), i.e., Neuroticism, Extraversion, Openness-to-Experience, Agreeableness and Conscientiousness. Items involve questions about typical behaviours or reactions, which are answered on a five-point Likert scale, ranging from "strongly disagree" to "strongly agree". The manual shows impressive indices of reliability and validity.

Dark Side Traits. Hogan Development Survey (HDS; Hogan \& Hogan, 1997) is a measure of the personality disorders expressed in non-clinical language. The survey includes 154 items, scored for 11 scales, each grouping 14 items. Respondents are requested to "agree” or "disagree” with the items. The HDS has been cross-validated with the MMPI personality disorder scales. It has considerable evidence of satisfactory reliability and validity (Fico, Hogan, \& Hogan, 2000; Hogan \& Hogan, 1999, 2001). Furnham \& Crump (2005) show the overlap of the HDS and DSM-IV disorder terminology. The HDS assesses dysfunctional interpersonal themes. It is argued that these dysfunctional dispositions reflect distorted beliefs about others that emerge when people encounter stress or stop considering how their actions affect others. Over time, these dispositions may become associated with a person's reputation and can impede job performance and career success. The HDS assesses self-defeating expressions of normal personality.

Value Preferences. The Motives, Values, Preferences Inventory (MVPI; Hogan \& Hogan, 1999) measures ten Motives/Preferences. Each scale is composed of five themes: a) Lifestyles, which concern the manner in which a 
person would like to live; b) Beliefs, which involve "shoulds", ideals and ultimate life goals; c) Occupational Preferences, which include the work an individual would like to do, what constitutes a good job, and preferred work materials; d) Aversions, which reflect attitudes and behaviours that are either disliked or distressing; and e) Preferred Associates, which include the kind of persons desired as co-workers and friends. The initial norms for the inventory were based on the responses of 10,000 employed adults from a variety of industries including health care, banking and finance, food service, construction and transportation. MVPI scores are quite stable over time, with test-retest reliabilities ranging between .64 and .88 (mean $=.79$ ). More than 100 validation studies have been conducted on the MVPI with results indicating that the inventory is effective in predicting job performance and outcome variables such as turnover.

\subsection{Procedure}

Participants were tested in an assessment centre and given feedback on their performance. Nearly all were native English speakers or else completely competent and confident in the language.

\section{Results}

\subsection{Personality Traits and Values}

All 221 participants' data was valid and used for analysis in the study. Table 1 shows the "raw" correlations between the five traits and the ten values. Four observations may be made. First, there was only one significant

Table 1. Correlations between the trait and values measure.

\begin{tabular}{|c|c|c|c|c|c|c|c|c|}
\hline & & & & $\mathrm{N}$ & E & $\mathrm{O}$ & A & C \\
\hline & & & $\mathrm{X}$ & 67.20 & 126.42 & 120.40 & 114.40 & 130.10 \\
\hline & & $\mathrm{X}$ & $\mathrm{SD}$ & 23.86 & 26.92 & 17.79 & 23.05 & 16.56 \\
\hline Recognition & $\begin{array}{l}\text { Desire to be known, seen, visible and famous, which leads to a } \\
\text { lifestyle guided by a search for opportunities to be noticed and } \\
\text { dreams of fame and high achievement, whether or not they are } \\
\text { fulfilled. }\end{array}$ & 40.48 & 7.66 & .02 & $.22^{* *}$ & $.19^{* *}$ & $-.28^{* *}$ & .05 \\
\hline Power & $\begin{array}{l}\text { Desire to succeed, make things happen, make a difference and } \\
\text { outperform the competition. }\end{array}$ & 46.39 & 6.56 & -.02 & $.24^{* * *}$ & .13 & $-.30^{* *}$ & $.14^{*}$ \\
\hline Hedonistic & $\begin{array}{l}\text { Pursuit of fun, excitement, pleasure and a lifestyle organised } \\
\text { around eating, drinking and entertainment. }\end{array}$ & 41.09 & 6.12 & .14 & $.16^{*}$ & .11 & -.11 & $-.13^{*}$ \\
\hline Altruistic & $\begin{array}{l}\text { Desire to help others, a concern for the welfare of the less } \\
\text { fortunate in life, and a lifestyle organised around public } \\
\text { service and the betterment of humanity. }\end{array}$ & 44.08 & 7.01 & .00 & .12 & $.25^{* *}$ & $.31^{* *}$ & .02 \\
\hline Affiliation & $\begin{array}{l}\text { Needing and enjoying frequent and varied social contact and a } \\
\text { lifestyle organised around social interaction. }\end{array}$ & 48.65 & 6.50 & -.02 & $.31^{* *}$ & $.28^{* *}$ & -.04 & .03 \\
\hline Tradition & $\begin{array}{l}\text { A belief in and dedication to old-fashioned virtues such as } \\
\text { family, church, thrift, hard work, appropriate social behaviour, } \\
\text { and a lifestyle that reflects these values. }\end{array}$ & 41.52 & 6.83 & .06 & .10 & .09 & .07 & $.17^{*}$ \\
\hline Security & $\begin{array}{l}\text { A need for predictability, structure and efforts to avoid risk } \\
\text { and uncertainty - especially in the employment area - and a } \\
\text { lifestyle organised around minimising errors and mistakes. }\end{array}$ & 36.22 & 6.83 & .01 & $-.20^{* *}$ & -.29 & $.15^{*}$ & $.27^{* *}$ \\
\hline Commerce & $\begin{array}{l}\text { Interest in earning money, realising profits, finding new } \\
\text { business opportunities, and a lifestyle organised around } \\
\text { investments and financial planning. }\end{array}$ & 44.29 & 6.53 & -.03 & .04 & -.02 & $-.18^{*}$ & $.30^{* *}$ \\
\hline Aesthetics & $\begin{array}{l}\text { Need for self-expression, a dedication to quality and } \\
\text { excellence, an interest in how things look, feel and sound and } \\
\text { close attention to the appearance of things. }\end{array}$ & 34.31 & 7.55 & $.15^{*}$ & .01 & $.49^{* *}$ & -.06 & $-.19^{* *}$ \\
\hline Science & $\begin{array}{l}\text { Being interested in science, comfortable with technology, } \\
\text { preferring data based - as opposed to intuitive decisions, and } \\
\text { spending time learning how things work. }\end{array}$ & 40.81 & 7.91 & .00 & -.12 & .07 & .02 & .04 \\
\hline
\end{tabular}


correlation with the trait Neuroticism and one correlation with the value Tradition and one with Science. Second, the trait of Conscientiousness seemed most closely linked with the values being significantly correlated with six, though the highest correlations were with Openness. Third, the value that seemed related to most of the traits was Security followed closely by Power. Finally, the size of the significant correlations were modest with only four of the fifty being $r>$.30. This suggests only a modest overlap between these two measures.

Table 2 shows the results of ten regressions with values as criterion variables and the Big Five traits the predictor variables. These results show three things. First, the Big Five accounted for most variance in Aesthetics, Altruism and Security and least for Science, Tradition and Hedonism. Second, Conscientiousness seemed surprisingly a significant predictor in only four values, while Agreeableness was a significant predictor in eight. This is similar to the findings from Olson and Weber (2004) where Agreeableness too was the most consistent significant predictor of value preference. Third, four of the five traits were significant predictors of security. Some regressions showed similar results. Thus, high emotionally stable extraverts favoured recognition and power. Low emotionally stable, agreeable, high conscientious people favoured tradition and security. Openness seemed strongly related to Aesthetics.

Table 3 shows the results of the factor analysis of the ten values, accounting for nearly three quarters of the

Table 2. Regressions of the Big Five onto each of the values/preference.

\begin{tabular}{|c|c|c|c|c|c|c|c|c|c|c|}
\hline & \multicolumn{2}{|c|}{ Recognition } & \multicolumn{2}{|c|}{ Power } & \multicolumn{2}{|c|}{ Hedonistic } & \multicolumn{2}{|c|}{ Altruistic } & \multicolumn{2}{|c|}{ Affiliation } \\
\hline & Beta & $t$ & Beta & $t$ & Beta & $t$ & Beta & $t$ & Beta & $t$ \\
\hline $\mathrm{N}$ & -.16 & $1.95^{*}$ & -.17 & $2.21^{*}$ & .11 & 1.37 & .26 & $3.52^{* *}$ & -.01 & 0.15 \\
\hline E & .16 & $2.42^{* *}$ & .18 & $2.83^{* *}$ & .16 & $2.37^{* *}$ & .10 & 1.66 & .25 & $3.83^{* * *}$ \\
\hline $\mathrm{O}$ & .11 & 1.67 & .04 & 0.63 & .04 & .54 & .51 & $4.74^{* * *}$ & .23 & $3.37^{* * *}$ \\
\hline A & -.34 & $4.42^{* * *}$ & -.38 & $5.11^{* * *}$ & -.02 & .24 & .51 & $7.05^{* * *}$ & .01 & .81 \\
\hline $\mathrm{C}$ & .03 & .46 & .11 & 1.74 & -.10 & 1.45 & .01 & 1.14 & .03 & .50 \\
\hline$F(5,220)$ & \multicolumn{2}{|c|}{$7.62^{* * *}$} & \multicolumn{2}{|c|}{$9.49^{* * *}$} & \multicolumn{2}{|c|}{$2.83^{*}$} & \multicolumn{2}{|c|}{$13.92^{* * *}$} & \multicolumn{2}{|c|}{$7.91^{* * *}$} \\
\hline \multirow[t]{3}{*}{ Adjusted $\mathrm{R}^{2}$} & \multicolumn{2}{|c|}{.13} & \multicolumn{2}{|c|}{.18} & \multicolumn{2}{|c|}{.04} & \multicolumn{2}{|c|}{.23} & \multicolumn{2}{|c|}{.12} \\
\hline & \multicolumn{2}{|c|}{ Tradition } & \multicolumn{2}{|c|}{ Security } & \multicolumn{2}{|c|}{ Commerce } & \multicolumn{2}{|c|}{ Aesthetics } & \multicolumn{2}{|c|}{ Science } \\
\hline & Beta & $t$ & Beta & $t$ & Beta & $t$ & Beta & $t$ & Beta & $t$ \\
\hline $\mathrm{N}$ & .24 & $2.84^{* *}$ & .24 & $3.11^{* *}$ & -.06 & .75 & .08 & 1.06 & .02 & .24 \\
\hline E & .10 & 1.51 & -.19 & 1.84 & .02 & .24 & -.09 & 1.48 & -.06 & .79 \\
\hline $\mathrm{O}$ & .10 & 1.40 & -.23 & $3.54^{* * * *}$ & -.03 & .50 & .50 & $8.14^{* * *}$ & .09 & 1.30 \\
\hline A & .20 & $2.56^{* *}$ & .21 & $2.80^{* *}$ & -.24 & $3.13^{* *}$ & .09 & 1.25 & .04 & .46 \\
\hline $\mathrm{C}$ & .23 & $3.31^{* * * *}$ & .31 & $4.80^{* * * *}$ & .30 & $4.41^{* * *}$ & -.12 & $1.97^{*}$ & .05 & .71 \\
\hline$F(5,220)$ & \multicolumn{2}{|c|}{$4.09^{* * *}$} & \multicolumn{2}{|c|}{$11.26^{* * * *}$} & \multicolumn{2}{|c|}{$6.61^{* * * *}$} & \multicolumn{2}{|c|}{$16.04^{* * *}$} & \multicolumn{2}{|c|}{$.48^{* * * *}$} \\
\hline Adjusted $\mathrm{R}^{2}$ & \multicolumn{2}{|c|}{.07} & \multicolumn{2}{|c|}{.19} & \multicolumn{2}{|c|}{.11} & \multicolumn{2}{|c|}{.26} & \multicolumn{2}{|c|}{.00} \\
\hline
\end{tabular}

Note. ${ }^{* * *} p<.001,{ }^{* *} p<.01,{ }^{*} p<.05$.

Table 3. Varimax rotated factor analyses of the ten value scales.

\begin{tabular}{|c|c|c|c|c|c|c|}
\hline & \multirow{2}{*}{ Variables } & \multirow{2}{*}{ Commonalities } & \multicolumn{4}{|c|}{ Factor } \\
\hline & & & 1 & 2 & 3 & 4 \\
\hline 1 & Commerce & .76 & .81 & .11 & .07 & -.28 \\
\hline 2 & Power & .74 & .76 & .02 & .36 & .18 \\
\hline 3 & Recognition & .70 & .64 & -.04 & .42 & .33 \\
\hline 4 & Science & .52 & .52 & .47 & -.14 & .05 \\
\hline 5 & Altruism & .82 & -.15 & .82 & .34 & .09 \\
\hline 6 & Tradition & .68 & .16 & .81 & .00 & .04 \\
\hline 7 & Affiliation & .79 & .10 & .20 & .85 & .05 \\
\hline 8 & Hedonism & .66 & .21 & -.04 & .79 & .00 \\
\hline 9 & Aesthetics & .78 & .10 & .35 & -.06 & .80 \\
\hline \multirow[t]{3}{*}{10} & Security & .62 & .16 & .43 & -.19 & -.61 \\
\hline & Eigenvalue & & 2.91 & 1.72 & 1.40 & 1.02 \\
\hline & Variance & & 29.17 & 17.11 & 14.01 & 10.29 \\
\hline
\end{tabular}


variance. The first factor was enterprising work values, while the second factor more associated traditional service values and the third with socialising work values. The final factor seemed more related to creativity and the life of the mind and could be labelled Non-Conformist work values. This analysis is very similar to that found in the MVPI Manual and serves as a replication. Whilst this factor analysis did not completely replicate the principal components analysis shown in the manual (Hogan \& Hogan, 1997: p. 18) there was sufficient overlap to make both interpretable. Following this four regressions were performed with the four value factors as criterion variable and the Big Five traits as predictor variables.

Table 4 indicates that all regressions were significant but showed very different patterns. The first regression indicated that low agreeable, conscientious people endorsed enterprising work values. The second regression indicated that highly agreeable, open, emotionally stable, conscientious individuals endorsed traditional service values. The third regression indicated that extraverted, open people endorsed socialising work values and the final regression that highly open, low conscientious people endorse values associated with non-conformist work values.

\subsection{Dark Side Traits and Values}

Table 5 shows the pattern of correlation between the ten values and eleven dark side traits. Two (Volatile, Passive Aggressive) seem weakly related to most of the values while others like Manipulative and Dramatic showed similar and strong associations. Three values (Tradition, Commerce, Science) seem weakly related to dark side traits.

Table 4. Regressions of the Big Five into the four factor scores.

\begin{tabular}{|c|c|c|c|c|c|c|c|c|}
\hline & \multicolumn{2}{|c|}{ Enterprising Work Values } & \multicolumn{2}{|c|}{ Traditional Service Values } & \multicolumn{2}{|c|}{ Socialising Work Values } & \multicolumn{2}{|c|}{ Non conformist work values } \\
\hline & Beta & $t$ & Beta & $t$ & Beta & $t$ & Beta & $t$ \\
\hline $\mathrm{N}$ & -.12 & 1.53 & .22 & $2.72^{* *}$ & .06 & .07 & -.09 & 1.35 \\
\hline $\mathrm{E}$ & .10 & 1.51 & .06 & .89 & .24 & $3.58^{* * * *}$ & .00 & .15 \\
\hline $\mathrm{O}$ & .08 & 1.18 & .21 & $3.14^{* *}$ & .16 & $2.27^{*}$ & .50 & $8.69^{* * *}$ \\
\hline A & -.31 & $3.99^{* * *}$ & .32 & $4.12^{* * *}$ & .00 & .09 & .06 & .09 \\
\hline $\mathrm{C}$ & .16 & $2.39^{* *}$ & .15 & $2.22^{*}$ & -.04 & .50 & -.28 & $4.85^{* * *}$ \\
\hline$F(5,220)$ & \multicolumn{2}{|c|}{6.46} & \multicolumn{2}{|c|}{5.71} & \multicolumn{2}{|c|}{5.16} & \multicolumn{2}{|c|}{23.64} \\
\hline Adjusted $\mathrm{R}^{2}$ & \multicolumn{2}{|c|}{.11} & \multicolumn{2}{|c|}{.10} & \multicolumn{2}{|c|}{.09} & \multicolumn{2}{|c|}{.34} \\
\hline
\end{tabular}

Note. ${ }^{* * *} p<.001,{ }^{* *} p<.01,{ }^{*} p<.05$.

Table 5. Correlations between the eleven dark side traits and ten values.

\begin{tabular}{|c|c|c|c|c|c|c|c|c|c|c|c|c|c|}
\hline & & \multirow{2}{*}{$\mathrm{X}$} & \multirow{2}{*}{ SD } & \multicolumn{10}{|c|}{ Values } \\
\hline & & & & 1 & 2 & 3 & 4 & 5 & 6 & 7 & 8 & 9 & 10 \\
\hline 1 & Excitable & 3.01 & 2.44 & .12 & .04 & $.14^{*}$ & -.07 & $-.14^{*}$ & .00 & .02 & -.09 & $.19^{* *}$ & -.04 \\
\hline 2 & Sceptical & 4.44 & 2.36 & $.37^{* *}$ & $.27^{* *}$ & $.22^{* *}$ & $-.24^{* *}$ & .03 & .09 & -.02 & .12 & .11 & .05 \\
\hline 3 & Cautious & 3.46 & 2.39 & $-.20^{* *}$ & $-.24^{* *}$ & .03 & -.05 & $-.35^{* *}$ & .02 & $.25^{*}$ & -.19 & .09 & .00 \\
\hline 4 & Reserved & 4.03 & 2.27 & -.12 & -.06 & -.08 & $-.27^{* *}$ & $-.36^{* *}$ & .00 & .12 & -.06 & .10 & .06 \\
\hline 5 & Leisurely & 4.91 & 1.93 & .12 & .09 & $.15^{*}$ & -.06 & .04 & .06 & .09 & .06 & .06 & .03 \\
\hline 6 & Bold & 7.10 & 2.52 & $.47^{* * *}$ & $.48^{* * *}$ & $.24^{* * *}$ & -.06 & .16 & -.04 & -.16 & $.18^{*}$ & $.14^{*}$ & .00 \\
\hline 7 & Mischievous & 7.15 & 2.44 & $.44^{* * *}$ & $.35^{* *}$ & $.26^{* *}$ & .01 & $.26^{* *}$ & -.06 & $-.39^{* * * *}$ & .06 & .13 & .07 \\
\hline 8 & Colourful & 7.81 & 2.95 & $.54^{* *}$ & $.37^{* *}$ & $.35^{* *}$ & .05 & $.43^{* * * *}$ & -.03 & $-.45^{* * *}$ & .05 & .13 & .06 \\
\hline 9 & Imaginative & 5.47 & 2.39 & $.37^{* * * *}$ & $.36^{* * *}$ & $.25^{* *}$ & $.15^{*}$ & $.20^{* *}$ & .13 & -.16 & $.15^{*}$ & $.30^{* *}$ & $.14^{*}$ \\
\hline 10 & Diligent & 8.90 & 3.46 & -.06 & .00 & -.07 & .08 & -.04 & .10 & $.36^{* *}$ & .14 & $-.18^{* *}$ & .10 \\
\hline 11 & Dutiful & 6.91 & 2.04 & .05 & $-.25^{* *}$ & -.09 & $.27^{* *}$ & .03 & $.17^{*}$ & $.20^{* *}$ & -.07 & .06 & .07 \\
\hline+12 & Social Desirability & 5.10 & 3.21 & .08 & .09 & .09 & .00 & -.08 & .06 & .07 & .08 & .00 & .11 \\
\hline
\end{tabular}

Note. ${ }^{* * *} p<.001,{ }^{* *} p<.01,{ }^{*} p<.05 .+$ Social Desirability was used in the analysis to check for the possibility of dissimulation. $1=$ Recognition, $2=$ Power, 3 = Hedonistic, 4 = Altruistic, 5 = Affiliation, $6=$ Tradition, $7=$ Security, $8=$ Commerce, $9=$ Aesthetics, $10=$ Science. 
Table 6 shows results of the ten regressions of which eight are significant. In most of these regressions it seemed one or two disorders were the only significant predictors. Thus, Reserved (Schizoid) was a strong (negative) predictor of Affiliation; Colourful of Hedonistic; Bold of Power; Imaginative of Aesthetics. Overall Excitable predicted modestly one value (Recognition), Sceptical two (Recognition and Altruistic), Cautious two (Power and Commerce), Reserved two (Altruistic and Affiliation) Leisurely one (Hedonistic), Bold two (Recognition and Power), Mischievous one (Security) and Colourful three (Recognition, Hedonism, Security).

Four of the regressions showed the dark side traits accounted for more than a quarter of the variance. Four dark side traits predicted valuing Recognition: Mischievous, Bold, Sceptical and Excitable types. Reserved types certainly did not value Affiliation but mischievous types did value Affiliation and these accounted for $40 \%$ of the variance. Bold, Dutiful, non-cautious types valued Power but no doubt for different reasons and this accounted for $30 \%$ of the variance. Around the same per cent of the variance was accounted for in the valuing of security. The Diligent and Dutiful did but the Mischievous and the Colourful did not value Security.

Table 7 shows the results of the final regressions. Once again the criterion variables were the ten different

Table 6. Regressions of the ten dark side traits (predictor variables) onto the ten values (criterion variables).

\begin{tabular}{|c|c|c|c|c|c|c|c|c|c|c|c|}
\hline & & \multicolumn{2}{|c|}{ Recognition } & \multicolumn{2}{|c|}{ Power } & \multicolumn{2}{|c|}{ Hedonistic } & \multicolumn{2}{|c|}{ Altruistic } & \multicolumn{2}{|c|}{ Affiliation } \\
\hline & & Beta & $t$ & Beta & $t$ & Beta & $t$ & Beta & $t$ & Beta & $t$ \\
\hline 1 & Excitable & .13 & $1.93^{*}$ & .12 & 1.70 & .13 & 1.68 & .08 & 1.09 & .03 & .48 \\
\hline 2 & Sceptical & .14 & $2.17^{*}$ & .08 & .23 & .05 & .62 & -.25 & $3.14^{* *}$ & .02 & .22 \\
\hline 3 & Cautious & -.09 & 1.36 & -.16 & $2.08^{*}$ & .08 & .89 & -.03 & .29 & -.07 & .98 \\
\hline 4 & Reserved & .00 & .76 & .0 & .00 & -.10 & 1.32 & -.23 & $3.16^{* *}$ & -.51 & $8.07^{* * *}$ \\
\hline 5 & Leisurely & .08 & 1.29 & .08 & 1.23 & .14 & $1.95^{*}$ & -.06 & .84 & .04 & .71 \\
\hline 6 & Bold & .18 & $2.61^{*}$ & .29 & $3.89^{* * * *}$ & .00 & .12 & .00 & .48 & .02 & .21 \\
\hline 7 & Mischievous & .11 & 1.58 & .07 & .88 & .03 & .41 & .05 & .64 & .02 & .22 \\
\hline 8 & Colourful & .36 & $4.89^{* * * *}$ & .13 & 1.65 & .29 & $3.30^{* * *}$ & -.08 & .88 & .22 & 2.93 \\
\hline 9 & Imaginative & .07 & 1.77 & .11 & 1.66 & .09 & 1.24 & .25 & $3.38^{* * *}$ & .09 & 1.50 \\
\hline 10 & Diligent & .09 & 1.55 & .09 & 1.49 & .03 & .37 & .07 & 1.05 & .01 & .24 \\
\hline 11 & Dutiful & .07 & 1.09 & -.15 & $1.91^{*}$ & -.11 & 1.50 & .24 & $3.35^{* * *}$ & -.01 & .18 \\
\hline \multicolumn{2}{|r|}{$F(11,2000)$} & \multicolumn{2}{|c|}{$14.18^{* * *}$} & \multicolumn{2}{|c|}{$9.21^{* * *}$} & \multicolumn{2}{|c|}{$4.49^{* *}$} & \multicolumn{2}{|c|}{$5.38^{* * *}$} & \multicolumn{2}{|c|}{$13.71^{* * * *}$} \\
\hline \multicolumn{2}{|r|}{ Adjusted $\mathrm{R}^{2}$} & \multicolumn{2}{|c|}{.41} & \multicolumn{2}{|c|}{.32} & \multicolumn{2}{|c|}{.15} & \multicolumn{2}{|c|}{.19} & \multicolumn{2}{|c|}{.40} \\
\hline & & \multicolumn{2}{|c|}{ Tradition } & \multicolumn{2}{|c|}{ Security } & \multicolumn{2}{|c|}{ Commerce } & \multicolumn{2}{|c|}{ Aesthetics } & \multicolumn{2}{|c|}{ Science } \\
\hline & & Beta & $t$ & Beta & $t$ & Beta & $t$ & Beta & $t$ & Beta & $t$ \\
\hline 1 & Excitable & .07 & .80 & .05 & 0.73 & -.03 & 0.35 & .14 & 1.80 & -.07 & .38 \\
\hline 2 & Sceptical & -.10 & 1.21 & .13 & 1.80 & .09 & 1.03 & -.03 & .31 & .08 & .89 \\
\hline 3 & Cautious & -.07 & .79 & .04 & .49 & -.22 & $2.42^{* *}$ & .06 & .66 & -.05 & .60 \\
\hline 4 & Reserved & .02 & .28 & .03 & .43 & .00 & .07 & .06 & .80 & .10 & 1.24 \\
\hline 5 & Leisurely & .04 & .47 & .01 & .16 & .07 & .91 & -.03 & .47 & -.02 & .31 \\
\hline 6 & Bold & -.02 & .21 & .02 & .28 & .09 & $1.38^{* *}$ & .04 & .54 & -.06 & .67 \\
\hline 7 & Mischievous & -.05 & .61 & -.20 & $2.68^{* *}$ & -.03 & .38 & .01 & .14 & .13 & 1.48 \\
\hline 8 & Colourful & -.03 & .32 & -.29 & $3.57^{* * *}$ & -.08 & .82 & .00 & .50 & -.15 & 1.60 \\
\hline 9 & Imaginative & .21 & $2.57^{* *}$ & .04 & .53 & .09 & 1.18 & .29 & $3.77^{* * * *}$ & .17 & 2.13 \\
\hline 10 & Diligent & .10 & 1.40 & .24 & $3.98^{* * *}$ & .15 & $2.11^{*}$ & -.15 & $2.23^{*}$ & .11 & 1.46 \\
\hline 11 & Dutiful & .16 & $2.12^{*}$ & .15 & $2.14^{*}$ & .00 & .10 & .10 & 1.31 & .13 & 1.60 \\
\hline \multicolumn{2}{|r|}{$F(11,2000)$} & \multicolumn{2}{|c|}{$1.59^{* * *}$} & \multicolumn{2}{|c|}{$8.66^{* * *}$} & \multicolumn{2}{|c|}{$2.19^{* * *}$} & \multicolumn{2}{|c|}{$3.51^{* *}$} & \multicolumn{2}{|c|}{$1.47^{* * * *}$} \\
\hline & Adjusted $\mathrm{R}^{2}$ & & 3 & & & & & & & & \\
\hline
\end{tabular}

Note. ${ }^{* * *} p<.001,{ }^{* *} p<.01,{ }^{*} p<.05$. 
Table 7. Regressions of the two blocks of variables and the incremental validity.

\begin{tabular}{|c|c|c|c|c|c|c|c|c|c|c|c|}
\hline & & \multicolumn{2}{|c|}{ Recognition } & \multicolumn{2}{|c|}{ Power } & \multicolumn{2}{|c|}{ Hedonistic } & \multicolumn{2}{|c|}{ Altruistic } & \multicolumn{2}{|c|}{ Affiliation } \\
\hline & & Beta & $t$ & Beta & $t$ & Beta & $t$ & Beta & $t$ & Beta & $t$ \\
\hline & $\mathrm{N}$ & -.02 & .21 & .01 & 0.13 & .24 & $2.42^{* * *}$ & .27 & $2.98^{* *}$ & .11 & 1.29 \\
\hline & E & .00 & .08 & .10 & 1.49 & .04 & .66 & .03 & .74 & .05 & .77 \\
\hline & $\mathrm{O}$ & .00 & .04 & -.07 & .98 & -.06 & .81 & .22 & $3.34^{* * *}$ & .10 & 1.60 \\
\hline & A & -.13 & 1.61 & -.16 & 1.83 & -.18 & $1.93^{*}$ & .48 & $5.59^{* * * *}$ & .10 & 1.25 \\
\hline & C & .08 & 1.27 & .10 & 1.38 & -.07 & .86 & .02 & .34 & .00 & .10 \\
\hline 1 & Excitable & .14 & $2.07^{*}$ & .14 & 1.86 & .06 & .71 & .01 & .13 & .00 & .06 \\
\hline 2 & Sceptical & .12 & 1.72 & .00 & .12 & .09 & 1.07 & -.14 & 1.84 & .04 & .63 \\
\hline 3 & Cautious & -.07 & .98 & -.14 & 1.72 & .00 & .06 & -.12 & 1.51 & -.10 & 1.27 \\
\hline 4 & Reserved & .00 & .07 & .00 & .24 & .07 & .83 & -.11 & 1.62 & -.47 & $7.01^{* * * *}$ \\
\hline 5 & Leisurely & .08 & 1.42 & .08 & 1.33 & .13 & 1.82 & -.06 & 1.02 & .04 & .70 \\
\hline 6 & Bold & .15 & $2.12^{*}$ & .25 & $3.27^{* * * *}$ & .06 & .71 & .01 & .18 & .00 & .07 \\
\hline 7 & Mischievous & .10 & 1.48 & .06 & .81 & .05 & .65 & .08 & 1.11 & .01 & .25 \\
\hline 8 & Colourful & .37 & $4.76^{* * * *}$ & .11 & 1.36 & .29 & $3.15^{* *}$ & -.08 & .95 & .20 & $2.62^{* *}$ \\
\hline 9 & Imaginative & .07 & 1.00 & .13 & 1.83 & .09 & 1.19 & .19 & $2.63^{* *}$ & .06 & .88 \\
\hline 10 & Diligent & .06 & 1.00 & .06 & .91 & .05 & .66 & .08 & 1.31 & .02 & .37 \\
\hline 11 & Dutiful & .08 & 1.32 & -.11 & 1.62 & -.12 & 1.72 & .19 & $2.82^{* *}$ & .03 & .40 \\
\hline & $F(16,211)$ & \multicolumn{2}{|c|}{$10.12^{* * * *}$} & \multicolumn{2}{|c|}{$7.16^{* * *}$} & \multicolumn{2}{|c|}{$3.66^{* * *}$} & \multicolumn{2}{|c|}{$7.01^{* * *}$} & \multicolumn{2}{|c|}{$9.81^{* * *}$} \\
\hline & 1 Adjusted $\mathrm{R}^{2}$ & \multicolumn{2}{|c|}{.14} & \multicolumn{2}{|c|}{.18} & \multicolumn{2}{|c|}{.04} & \multicolumn{2}{|c|}{.24} & \multicolumn{2}{|c|}{.13} \\
\hline & 2 Adjusted $\mathrm{R}^{2}$ & \multicolumn{2}{|c|}{$.41^{*}$} & \multicolumn{2}{|c|}{.32} & \multicolumn{2}{|c|}{.17} & \multicolumn{2}{|c|}{.31} & \multicolumn{2}{|c|}{.40} \\
\hline
\end{tabular}

\begin{tabular}{|c|c|c|c|c|c|c|c|c|c|c|c|}
\hline & \multicolumn{2}{|c|}{ Tradition } & \multicolumn{2}{|c|}{ Security } & \multicolumn{2}{|c|}{ Commerce } & \multicolumn{2}{|c|}{ Aesthetics } & \multicolumn{2}{|c|}{ Science } \\
\hline & & Beta & $t$ & Beta & $t$ & Beta & $t$ & Beta & $t$ & Beta & $t$ \\
\hline & $\mathrm{N}$ & .21 & $1.99^{*}$ & .04 & .37 & .04 & .39 & .07 & .76 & .07 & .63 \\
\hline & E & .13 & 1.67 & .03 & .42 & .00 & .04 & -.06 & 0.90 & .01 & .19 \\
\hline & $\mathrm{O}$ & .09 & 1.13 & -.17 & $2.54^{* *}$ & -.04 & .52 & .48 & $7.11^{* * *}$ & .04 & .52 \\
\hline & A & .18 & 1.83 & .04 & .47 & -.11 & 1.11 & .12 & 1.35 & .11 & 1.01 \\
\hline & C & .26 & $3.27^{* * *}$ & .18 & $2.67^{* *}$ & .25 & $3.08^{* *}$ & -.04 & .60 & .03 & .27 \\
\hline 1 & Excitable & .06 & .73 & .00 & .08 & .01 & .12 & .08 & .99 & -.09 & .99 \\
\hline 2 & Sceptical & -.03 & .64 & .13 & 1.81 & .06 & .71 & .02 & .19 & .10 & 1.09 \\
\hline 3 & Cautious & -.12 & 1.25 & .02 & .26 & -.21 & $2.22^{*}$ & .05 & .65 & -.08 & .82 \\
\hline 4 & Reserved & .12 & 1.48 & .04 & .51 & .01 & .12 & .14 & 1.88 & .12 & 1.44 \\
\hline 5 & Leisurely & .04 & .58 & .01 & .19 & .08 & 1.13 & -.02 & .34 & -.03 & .31 \\
\hline 6 & Bold & -.09 & .99 & -04 & .48 & .05 & .55 & .07 & .94 & -.06 & .60 \\
\hline 7 & Mischievous & -.02 & .28 & -.17 & $2.30^{*}$ & -.02 & .23 & -.01 & .19 & .14 & 1.59 \\
\hline 8 & Colourful & -.03 & .35 & -.26 & $3.18^{* *}$ & -.05 & .52 & -.01 & .11 & -.14 & 1.42 \\
\hline 9 & Imaginative & .19 & $2.66^{* *}$ & .11 & 1.52 & .11 & 1.38 & .13 & 1.75 & .16 & 1.87 \\
\hline 10 & Diligent & .03 & 0.43 & .18 & $2.86^{* *}$ & .07 & .96 & -.12 & 1.77 & .10 & 1.33 \\
\hline 11 & Dutiful & .15 & $2.01^{*}$ & .15 & $2.34^{*}$ & .03 & .37 & .07 & 1.07 & .12 & 1.41 \\
\hline & $F(16,211)$ & \multicolumn{2}{|c|}{$2.31^{*}$} & \multicolumn{2}{|c|}{$7.18^{* * * *}$} & \multicolumn{2}{|c|}{$2.22^{* *}$} & \multicolumn{2}{|c|}{$6.37^{* *}$} & \multicolumn{2}{|c|}{1.08} \\
\hline & 1 Adjusted $\mathrm{R}^{2}$ & \multicolumn{2}{|c|}{.07} & \multicolumn{2}{|c|}{.19} & \multicolumn{2}{|c|}{.11} & \multicolumn{2}{|c|}{.26} & \multicolumn{2}{|c|}{.00} \\
\hline & 2 Adjusted $\mathrm{R}^{2}$ & \multicolumn{2}{|c|}{.09} & \multicolumn{2}{|c|}{.32} & \multicolumn{2}{|c|}{.16} & \multicolumn{2}{|c|}{.29} & \multicolumn{2}{|c|}{.00} \\
\hline
\end{tabular}

Note. ${ }^{* * *} p<.001,{ }^{* *} p<.01,{ }^{*} p<.05$. 
values. In this analysis two blocks of variables were entered as the predictor variables. First, the Big Five personality traits were entered, then the eleven dark side traits were entered. Table 7 shows the results when both blocks were entered but also the incremental variance accounted for by the second block, which was the focus of this analysis. Thus the adjusted $\mathrm{R}^{2}$ is given first for the Big Five, then for the Big Five and the Dark Side Traits.

Six of the regressions were significant at $p<.001$. For Recognition, the Big Five accounted for $14 \%$ of the variance but when the dark side traits were added this increased to $41 \%$. Colourful and to a lesser extent Excitable and Bold predicted this value. For Power the variance increased from $8 \%$ to $32 \%$ the only significant predictor being Bold. Hedonism has three significant predictors and the variance increased from $3 \%$ to $17 \%$. Bold, neurotic, disagreeable types favoured the pursuit of Hedonism. The variances accounted for in the preference for Altruism increased from $24 \%$ to $31 \%$ and three traits and two disorders were significant predictors. Dutiful, Imaginative, Agreeable, Open, Unstable (Neurotic) individuals clearly favoured this value. For the value Affiliation the variance increased from $13 \%$ to $40 \%$ once disorders were added to the traits. Colourful, low reserved individuals rated this value as important. Finally there were six significant predictors (two traits, four disorders) for the value Security which saw an incremental validity of $14 \%$ (18\% - 32\%) once disorders were added to the traits. Low Conscientious, Neurotic, Imaginative and Dutiful (Dependent) people rated Tradition highly. It is also interesting to note that the values of Aesthetics and Science had no significant predictors and Commerce only one which was Cautiousness.

\section{Discussion}

This study looked at the relationship between three individual difference variables: bright side traits, dark side traits and values, which made-up the three parts of the socio-analytic understanding of the individual (Hogan, 2006). The types of values people hold no doubt predict both where and how they work. Values, along with traits abilities and dark side traits influence what sort of jobs and organisations people seek out but also how they work in them.

If one examines the factor analysis (Table 3) of the HDS (Furnham \& Crump, 2005), it may be possible to discern logical business types or clusters. Thus, the one factor indicates individuals very much concerned with operational variables, systems and task (Enterprising Work Values). Another indicates a more interpersonal, consultative, socio-emotional approach to work (Socialising Work Values). A third may be concerned with creativity and innovation (non-conformist work values) and a fourth with Security, Tradition and Continuity (traditional service values). Thus, a factor or cluster analysis of all three instruments simultaneously, may help to understand distinct business people typologies as well as how and why they chose to operate as they did.

What seemed clear from the final analysis (Table 7) is that most of the values have very clear bright and dark side traits. Some values like Altruism and Security seem particularly linked to multiple traits while others like Power or Aesthetics seem linked to only one or other trait. Thus, a high Power score is a good "marker" for the Bold (or Narcissistic disorder) while Aesthetics is a good "marker" for Openness-to-Experience. Thus, a profile with unique and high scores on certain values may be particularly useful in delineating bright and dark side trait differences. This maybe particularly useful in certain situations where people or organisations dislike or distrust measuring personality traits. Further it is possible that value measures are less open to dissimulation and impression management bias than trait measures.

Looking at the relationship between traits and values, it is apparent that this study replicates others in the area. Thus relatively few of the values are related to Neuroticism (Costa et al., 1984), that Openness most powerfully relates to Artistic/Aesthetic values (De Fruyt \& Mervielde, 1997) and that Conscientiousness best predicts values in Commerce and Enterprise (De Fruyt \& Mervielde, 1997).

The correlations however are modest though none essentially counterintuitive. The results of the regressions show that only two values (Altruism and Aesthetics) are moderately related to the five personality variables in that the latter accounts for more than a fifth of the variance. However, the results make good sense. Conscientious individuals value Tradition, Security and Commerce but eschew Aesthetics. They like order and structure and have high need for achievement. They tend to be intellectually and attitudinally conservative and their educational and job preferences reflect that. Agreeable people, on the other hand, value Tradition and Security but are do not value Commerce or the drivers of enterprise: Recognition and Power. They do, of course, value Altruism which is a major facet of it.

Socialisation may accentuate the links between traits and values. That is, over time, traits become more clearly related to particular values that reflect the skills, preferences and goals of individuals with particular pro- 
files. Thus, trait agreeable individuals find it easier to be altruistic, which they learn to value, but they find competition for Power and Recognition much less attractive. Equally, individuals highly Open-to-Experience naturally find all aspects of Aesthetics appealing given their particular skill set. Indeed Openness is one of the best predictors of art preference, knowledge and consumption (Furnham, 2007).

It is interesting that some more obviously vocational values seem much less related to personality traits. Thus there are no significant predictors or correlates of science. This may mean that people with any temperament may be attracted to, or socialised into, have an interest in, and have values associated with, science-like observation and experiment. On the other hand it could well be cognitive ability (measured by power tests) rather than personality (measured by preference tests) that is more closely related to valuing the scientific methods and approach, as well as being successful in "scientific occupations".

Although a case could be made for consciously socialising nearly all of the values into individuals in schools, families and work places, only one-namely Hedonism—seems less desirable. Extraversion is modestly related to Hedonism with its association with excitement. Power too may seem less attractive though recognized for many years as an important individual need. Indeed, the cross-cultural even within-cultural, differences in ratings of the virtues and associated desirability of these values may be great. Thus, while some may believe it is important to socialize the values of Security and Tradition, others may strongly endeavour to ensure these values are not transmitted from the generation to the next.

Perhaps the most unique and important part of this study is to be seen in Table 6 and Table 7 referring to the dark side traits. Table 7 shows the incremental validity of the dark over the bright side personality traits in predicting values. For six of the values a combination of bright and dark side traits accounted for around $30 \%$ of the variance or more. Further the incremental validity was over $10 \%$ for Power, Hedonism and Security and over 20\% for Recognition and Affiliation. Table 7 showed that particular individual disorders were strongly, clearly and uniquely related to particular values. Thus, Colourful (Histrionic) related powerfully to valuing Recognition; Bold (Narcissistic) Power and Reserved low Affiliation. Overall the dark side traits were weakly related to valuing Science, Aesthetics, Commerce and Tradition.

Thus, powerful endorsement of some values (i.e., commercial) may note a management-derailing tendency in terms of dark side traits. However, this needs to be verified by future investigations, which would benefit from having various reliable and valid behavioural measures of work or educational success as dependent variables. Thus, it may be possible to test the incremental validity of the different measures in predicting real world outcomes.

The modest correlations between these three self-report measures may be contrary to the expectations of both researchers and recruiters. They have unique and incremental validity and therefore it could be argued all three need to be used in research and assessment depending on the needs and objectives of assessors (Hogan \& Hogan, 1999). However, it must be acknowledged that this study would have been strengthened by having an external valid criterion measure such as one associated with work productivity, learning ability in training or supervisor ratings. This would show the differential ability of these three measures to predict non-self report behavioural measures.

It appears that no one has studied the links between these three inventories of very different conceptual domains. Because all three inventories are used worldwide for coaching and career guidance, the data will be relevant for both academics and practitioners. What we discover is that, in general, they are only loosely related, which means all three are needed for individual assessment. But the exception to this generalisation is the correlations between some values and some HDS scales.

This study has shown logical and consistent overlap between three self-report measures of rather different variables. This raises theoretical and measurement questions. The first is whether significant correlations are more a result of item or methodology overlap than conceptual similarity. It is generally agreed that multi-trait, multi-method research is best and that correlations between different types of measures (i.e., self-report vs. observation) is always lower than between measures of different constructs but with similar methods. However what is noticeable from the correlational (Table 1, Table 5) and regressional (Table 2, Table 4, Table 6, Table 7) results is that the overlap is not particularly high. Further, a content analysis of the questions from each measure looking at style content and response scale would suggest that there is inevitably some overlap. Certainly it would be desirable to use observational as well as self-report data to attempt to overcome methodological overlap. Whilst there are observer (i.e., spouse, partner) versions of trait measures as yet it seems there are no observer measures of values. However, a number of observer checklist of the personality disorders exist 
(Saulsman \& Page, 2004) and this may be a promising avenue of research.

Secondly, what are the implications for measurement? Whilst there is a fairly large literature on the relationship when personality and values and that these results replicate perhaps the most interesting results are the overlap between dark side traits and values. One question is to what extent may one expect the evidence of a personality disorder if a participant rates a value particularly highly? Thus, could a very high score on Recognition be evidence for, or perhaps a clue to, a Narcissistic personality (Colourful), or a very high score for Power be an indication of a psychopath (Bold)? Certainly it would be unwise to conclude that extreme value scores indicate dark side traits of some sort, but they probably should alert interviewers and selectors to the possibility of other extreme tendencies. In our consulting work, the authors regard very high scores on Commercial as a sign of greed, and very low scores on Altruism as a sign of selfishness.

Like all others this paper had various limitations. The most important was that it relied entirely on self-report which has well known problems associated with social desirability as well as self-awareness. It is always desirable to have other data sources such as observational data to supplement self-report data. Next, whilst the population for the study was sufficiently large for the analyses it would be desirable to have a larger, more heterogenous sample to test the hypotheses on.

Future studies in this area may benefit from examining various demographic factors associated with the participant's such as their work experience as well as their performance data. It is also desirable to get longitudinal data to see how people change their values and preferences over time. Equally future research may benefit from replicating these findings using a different values or preference measure

\section{References}

Babiak, P., \& Hare, R. (2006). Snakes in Suits. New York: Regan Books.

Costa, P. T., \& McCrae, R. R. (1992). Normal Personality Assessment in Clinical Practice: The NEO Personality Inventory. Psychological Assessment, 4, 5. http://dx.doi.org/10.1037/1040-3590.4.1.5

Costa, P., McCrae, R., \& Holland, J. (1984). Personality and Vocational Interests in an Adults Sample. Journal of Applied Psychology, 69, 390-400. http://dx.doi.org/10.1037/0021-9010.69.3.390

De Clercq, B., \& De Fruyt, F. (2003). Personality Disorder Symptoms in Adolescence: A Five-Factor Model Perspective. Journal of Personality Disorders, 17, 269-292. http://dx.doi.org/10.1521/pedi.17.4.269.23972

De Fruyt, F., \& Mervielde, I. (1997). The Five-Factor Model of Personality and Holland's RIASEC Interest Types. Personality and Individual Differences, 23, 87-103. http://dx.doi.org/10.1016/S0191-8869(97)00004-4

Dyce, J. (1997). The Big Five Factors of Personality and Their Relationships to Personality Disorders. Journal of Clinical Psychology, 53, 587-593. http://dx.doi.org/10.1002/(SICI)1097-4679(199710)53:6<587::AID-JCLP7>3.0.CO;2-H

Fico, J., Hogan, R., \& Hogan, J. (2000). Interpersonal Compass Manual and Interpretation Guide. Tulsa, OK: Hogan Assessment Systems.

Furnham, A. (2007). Personality Disorders and Derailment at Work. In J. Langan-Fox, C. Cooper \& R. Klimoski (Eds.). Management Challenges and Symptoms of the Dysfunctional Workplace. Cheltenham: Edward Elgar

Furnham, A., \& Crump, J. (2005). Personality Traits, Types and Disorders. European Journal of Personality, 19, $167-184$. http://dx.doi.org/10.1002/per.543

Furnham, A., \& Taylor, J. (2004). The Dark Side of Behaviour at Work. Basingstoke: Palgrave. http://dx.doi.org/10.1057/9780230510104

Furnham, A., Hyde, G., \& Trickey, G. (2013). The Values of Work Success. Personality and Individual Differences, 55, 485-490. http://dx.doi.org/10.1016/j.paid.2013.04.016

Furnham, A., Hyde, G., \& Trickey, G. (2014a). Do Your Dark Side Traits Fit? Applied Psychology, 63, 589-606. http://dx.doi.org/10.1111/apps.12002

Furnham, A., Hyde, G., \& Trickey, G. (2014b). The Dark Side of Career Preference. Journal of Applied Social Psychology, 44, 106-114. http://dx.doi.org/10.1111/jasp.12205

Gaddis, B., \& Foster, J. (2015). Meta-Analysis of Dark Side Personality Characteristics and Critical Work Behaviours among Leaders across the Globe. Applied Psychology, 64, 25-54. http://dx.doi.org/10.1111/apps.12017

Hogan, R. (2006). Personality and the Fate of Organisations. New York: Lawrence Erlbaum.

Hogan, R., \& Blake, R. (1999). John Holland's Vocational Typology and Personality Theory. Journal of Vocational Psychology, 55, 41-56. http://dx.doi.org/10.1006/jvbe.1999.1696

Hogan, R., \& Hogan, J. (1997). Hogan Development Survey Manual. Tulsa, OK: Hogan Assessment Centres. 
Hogan, R., \& Hogan, J. (1999). Motives, Values, Preferences Inventory Manual. Tulsa, OK: Hogan Assessment Centre.

Hogan, R., \& Hogan, J. (2001). Assessing Leadership: A View from the Dark Side. International Journal of Selection and Assessment, 9, 40-51. http://dx.doi.org/10.1111/1468-2389.00162

Judge, T. A., Piccolo, R. F., \& Kosalka, T. (2009). The Bright and Dark Sides of Leader Traits: A Review and Theoretical Extension of the Leader Trait Paradigm. The Leadership Quarterly, 20, 855-875. http://dx.doi.org/10.1016/j.leaqua.2009.09.004

Kaiser, R. B., \& Craig, S. B. (2011). Do the Behaviors Related to Managerial Effectiveness Really Change with Organizational Level? An Empirical Test. The Psychologist-Manager Journal, 14, 92-119. http://dx.doi.org/10.1080/10887156.2011.570140

Kaiser, R. B., Craig, S. B., Overfield, D. V., \& Yarborough, P. (2011). Differences in Managerial Jobs at the Bottom, Middle, and Top: A Review of Empirical Research. The Psychologist-Manager Journal, 14, 76-91. http://dx.doi.org/10.1080/10887156.2011.570137

Kaiser, R., Hogan, R., \& Craig, S. (2008). Leadership and the Fate of Organizations. American Psychologist, 63, 96-100. http://dx.doi.org/10.1037/0003-066X.63.2.96

Kets de Vries, M. (2006). The Leader on the Couch. Basingstoke: Palgrave MacMillan.

Kets de Vries, M. (2007). Coach and Couch: The Psychology of Making Better Leaders. Basingstoke: Palgrave MacMillan.

Kets de Vries, M., \& Miller, D. (1985). The Neurotic Organisation. San Francisco, CA: Jossey Bass.

McAdams, D. (1995). What Do We Know When We Know a Person? Journal of Personality, 63, 365-396. http://dx.doi.org/10.1111/j.1467-6494.1995.tb00500.x

Olson, K., \& Weber, D. (2004). Relations between Big Five Traits and Fundamental Motives. Psychological Reports, 95, 795-802.

Rolland, J.-P., \& De Fruyt, F. (2003). The Validity of FFM Personality Dimensions and Maladaptive Traits to Predict Negative Affect at Work: A Six Month Prospective Study in a Military Sample. European Journal of Personality, 17, S101-S121. http://dx.doi.org/10.1002/per.485

Saulsman, L., \& Page, A. (2004). The Five Factor Model and Personality Disorder Empirical Literature: A Meta-Analytic Review. Clinical Psychology Review, 23, 1055-1085. http://dx.doi.org/10.1016/j.cpr.2002.09.001

Stepp, S., \& Trull, T. (2007). Predictive Validity of the Five Factor Model Prototype Scores for Antisocial and Borderline Personality Disorders. Personality and Mental Health, 1, 27-39. http://dx.doi.org/10.1002/pmh.3

Widiger, R., \& Costa, P. T. (2001). Five Factor Model Personality Disorder Research. In P. T. Costa, \& T. A. Widiger (Eds.). Personality Disorders and the Five Factor Model of Personality (2nd ed., pp. 59-87). Washington DC: American Psychological Association.

Widiger, R., Costa, P. T. \& McCrae, R. R. (2001). Proposals for Axis II: Diagnosing Personality Disorders Using the Five Factor Model. In P. T. Costa, \& T. A. Widiger (Eds.), Personality Disorders and the Five Factor Model of Personality (2nd ed., pp. 432-456). Washington DC: American Psychological Association.

Widiger, T. A., Trull, T. J., Clarkin, J. F., Sanderson, C., \& Costa, P. T. (2002). A Description of the DSM-IV Personality Disorders with the Five-Factor Model of Personality. In P. T. Costa, \& T. A. Widiger (Eds.), Personality Disorders and the Five Factor Model of Personality (2nd ed., pp. 89-99). Washington DC: American Psychological Association. http://dx.doi.org/10.1037/10423-006

Widiger, T., \& Coker, L. (2001). Assessing Personality Disorders. In J. N. Butcher (Ed.), Clinical Personality Assessment: Practical Approaches (2nd ed., pp. 407-434). New York: Oxford University Press.

Wiggins, J., \& Pincus, A. (1989). Conceptions of Personality Disorders and Dimensions of Personality. Psychological Assessment, 1, 305-316. http://dx.doi.org/10.1037/1040-3590.1.4.305

Winter, D., John, O., Stewart, A., Klohnen, E., \& Duncan, L. (1998). Traits and Motives: Toward an Integration of Two Traditions in Personality Research. Psychological Review, 105, 230-250.

http://dx.doi.org/10.1037/0033-295X.105.2.230 